\title{
Direct observation of Josephson capacitance
}

\author{
M. A. Sillanpää ${ }^{1}$, T. Lehtinen ${ }^{1}$, A. Paila ${ }^{1}$, Yu. Makhlin ${ }^{1,2}$, L. Roschier ${ }^{1}$, and P. J. Hakonen ${ }^{1}$ \\ ${ }^{1}$ Low Temperature Laboratory, Helsinki University of Technology, FIN-02015 HUT, Finland \\ ${ }^{2}$ Landau Institute for Theoretical Physics, 119334 Moscow, Russia
}

\begin{abstract}
The effective capacitance has been measured in the split Cooper pair box (CPB) over its phase-gate bias plane. Our low-frequency reactive measurement scheme allows to probe purely the capacitive susceptibility due to the CPB band structure. The data are quantitatively explained using parameters determined independently by spectroscopic means. In addition, we show in practice that the method offers an efficient way to do non-demolition readout of the CPB quantum state.

PACS numbers: $67.57 . F g, 47.32 .-y$
\end{abstract}

Energy can be stored into Josephson junctions (JJ) according to $E=-E_{J} \cos (\varphi)$, where $\varphi$ is the phase difference across the junction, and the Josephson energy $E_{J}$ is related to the junction critical current $I_{C}$ through $I_{C}=2 e E_{J} / \hbar$. By using the Josephson equations, this energy storing property translates into the well-known fact that a single classical JJ behaves as a parametric inductance $L_{J}=\hbar /\left(2 e I_{C}\right)$ for small values of $\varphi$.

Since the early 80 's, it has become understood that $\varphi$ itself can behave as a quantum-mechanical degree of freedom [1]. In mesoscopic JJs, this is typically associated with the competition between the Josephson and Coulomb effects at a very low temperature. These fundamental phenomena take place if charge on the junction is localized by a large resistance $R>R_{Q}=h /\left(4 e^{2}\right)$ [2], as well as in the Cooper-pair box (CPB), or the singleCooper-pair transistor (SCPT), whose quantum coherence is often considered macroscopic [3] .

In the first theoretical landmark papers [4, 5] on quantum properties of $\varphi$ it was already noticed that due to localization of charge $Q$, the energy of a the JJ system is similar to that of a non-linear capacitance. In spite of the importance of the phenomenon especially in CPB or SCPT in the promising field of superconducting qubits [6, 7], direct experimental verification of the Josephson capacitance has been lacking, likely due to challenges posed by measuring small reactances, or by the extreme sensitivity to noise.

In this Letter, we present the first such direct experiment [8], where we determine the Josephson capacitance in the Cooper pair box. Related experiments have recently been performed by Wallraff et al. 9], but in their case the key role is played by the transitions between levels of a coupled system where the band gap between the ground state and first excited state of the CPB, $E_{1}-E_{0}=\Delta E$, is nearly at resonance with an oscillator of angular frequency $\omega_{0}$. Thus, detuning fully dominates over the Josephson capacitance which can be clearly observed in our experiments where we study directly the reactive response of the lowest band $E_{0}$ at a frequency $\omega_{0} \ll \Delta E / \hbar$. We determine the experimental parameters independently using spectroscopy, and demonstrate a simple way to perform a non-destructive measurement of the CPB state using purely the CPB Josephson capacitance.

An SCPT (Fig. 1) consists of a mesoscopic island (total capacitance $C=C_{1}+C_{2}+C_{g}$ ), two JJs, and of a nearby gate electrode used to polarize the island with the (reduced) gate charge $n_{g}=C_{g} V_{g} / e$. The island has the charging energy $E_{C}=e^{2} /(2 C)$, and the junctions have the generally unequal Josephson energies $E_{J}(1 \pm d)$, where the asymmetry is given by $d$. The SCPT Hamiltonian is then $E_{C}\left(\hat{\mathrm{n}}-n_{g}\right)^{2}-2 E_{J} \cos (\varphi / 2) \cos (\hat{\theta})+$ $2 d E_{J} \sin (\varphi / 2) \sin (\hat{\theta})-C_{g} V_{g}^{2} / 2$. Here, the number $\hat{n}$ of extra electron charges on the island is conjugate to $\hat{\theta} / 2$, where $\hat{\theta}$ is the superconducting phase on the island [10]. The SCPT is thus equivalent to a CPB (single JJ and a capacitance in series with a gate voltage source) but with a Josephson energy tunable by $\varphi=2 \pi \Phi / \Phi_{0}$, where $\Phi_{0}=h /(2 e)$ is the quantum of magnetic flux.

If $d=0$ and $E_{J} / E_{C} \ll 1$ the ground and excited state energies are $\left(n_{g}=0 \ldots 2\right): E_{0,1}=E_{C}\left(n_{g}^{2}-2 n_{g}+\right.$ $2) \mp \sqrt{\left(E_{J} \cos (\varphi / 2)\right)^{2}+\left(2 E_{C}\left(1-n_{g}\right)\right)^{2}}-C_{g} V_{g}^{2} / 2$, with a large gap to higher levels. For a general $E_{J} / E_{C}$, we compute the bands numerically in the charge state basis.

The effective "Josephson" capacitance of the CPB can be related to the curvature of band $k$, similar to the effective mass of an electron in a crystal:

$$
C_{\mathrm{eff}}^{k}=-\frac{\partial^{2} E_{k}\left(\varphi, n_{g}\right)}{\partial V_{g}^{2}}=-\frac{C_{g}^{2}}{e^{2}} \frac{\partial^{2} E_{k}\left(\varphi, n_{g}\right)}{\partial n_{g}^{2}} .
$$

Usually, the system effective capacitance is obtained from a Lagrangian or Hamiltonian as $\partial^{2} \mathcal{L} / \partial V_{g}^{2}=$ $\left(\partial^{2} H / \partial Q^{2}\right)^{-1}$, without the minus sign. In Eq. (1), however, $E_{k}$ 's are, more precisely, the eigenvalues of the Routhian $\mathcal{H}=\dot{\theta} \partial_{\dot{\theta}} \mathcal{L}-\mathcal{L}$ [1], which serves as a Hamiltonian for the $n, \theta$ degree of freedom but as minus Lagrangian for the phase $\alpha \equiv \frac{e}{\hbar} \int V_{g} d t$ and $V_{g} \propto \dot{\alpha}$, thus leading to Eq. (11).

Using the analytic formulas for $E_{0,1}$ in the limit 


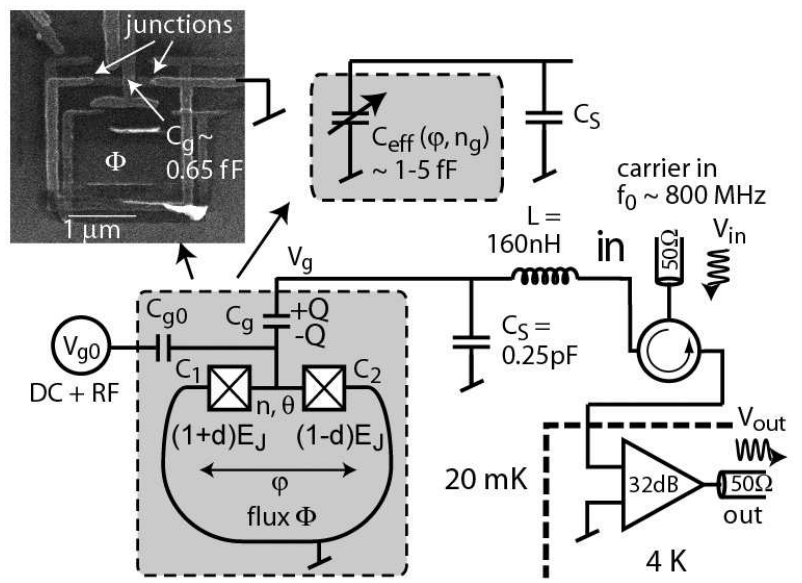

FIG. 1: Schematic view of the experiment. The resonant frequency of the $L C$ circuit (made using lumped elements) is tuned by the effective capacitance $C_{\text {eff }}$ of the Cooper pair box shown in the SEM image. For details, see text.

$$
\begin{aligned}
& E_{J} / E_{C} \ll 1 \text { we get } \\
& C_{\mathrm{eff}}^{(0,1)}=C_{g}-\frac{2 C_{g}^{2} E_{C}}{e^{2}} \times \\
& \times\left(1 \mp \frac{E_{C} E_{J}^{2}(1+\cos \varphi)}{\left[4 E_{C}^{2}\left(n_{g}-1\right)^{2}+\frac{1}{2} E_{J}^{2}(1+\cos \varphi)\right]^{3 / 2}}\right),
\end{aligned}
$$

which reduces to the classical geometric capacitance $\left(1 / C_{g}+1 /\left(C_{1}+C_{2}\right)\right)^{-1}$ in the limit of vanishingly small $E_{J}$, except where Cooper-pair tunneling is degenerate 12. Numerically evaluated graphs of $C_{\mathrm{eff}}^{(0,1)}$ for a general $E_{J} / E_{C}$ can be found in Ref. 13 .

Our experimental scheme is illustrated in Fig. 1 We perform low-dissipation microwave reflection measurements [14, 15] on a series $L C$ resonator in which the box effective capacitance, Eq. (10), is a part of the total capacitance $C_{S}+C_{\text {eff }}^{k}$. The resonator is formed by a surface mount inductor of $L=160 \mathrm{nH}$. With a stray capacitance of $C_{S}=250 \mathrm{fF}$ due to the fairly big lumped resonator, the resonant frequency is $f_{0}=800 \mathrm{MHz}$ and the quality factor $Q \simeq 16$ is limited by the external $Z_{0}=50 \Omega$. When $C_{\text {eff }}^{k}$ varies, the phase $\Theta$ of the reflected signal $V_{\text {out }}=\Gamma V_{\text {in }}$ changes, which is measured by the reflection coefficient $\Gamma=\left(Z-Z_{0}\right) /\left(Z+Z_{0}\right)=\Gamma_{0} e^{i \Theta}$. Here, $Z$ is the resonator impedance seen at the point labelled "in" in Fig. [1 In all the measurements, the probing signal $V_{\text {in }}$ was continuously applied.

Since we are rather far from matching conditions, the reflection magnitude $\Gamma_{0}$ remains always close to one. The variation in $\Theta$ due to modulation in $C_{\text {eff }}^{k}$ is up to $40^{\circ}$ in our measurements, corresponding to a shift of resonance frequency $\Delta f_{0} \simeq 6 \mathrm{MHz}$. In addition to band pass filtering, we used two circulators at $20 \mathrm{mK}$.

As seen in Eq. (21), the modulation depth of $C_{\mathrm{eff}}^{k}$ is sensitive to $C_{g}$. Therefore, in order to faithfully demonstrate the Josephson capacitance in spite of the stray capacitance, we used a large $C_{g}>0.5 \mathrm{fF}$. It was made using an $\mathrm{Al}-\mathrm{AlO}_{x}-\mathrm{Al}$ overlay structure (see the image in Fig. (1), with a prolonged oxidization in 0.1 bar of $\mathrm{O}_{2}$ for 15 min. Otherwise, our CPB circuits have been prepared using rather standard e-beam lithography. The tunnel junctions having both an area of $60 \mathrm{~nm} \times 30 \mathrm{~nm}$ correspond to an average capacitance of $\sim 0.17 \mathrm{fF}$ each. The overlay gate has $C_{g} \simeq 0.7 \mathrm{fF}$ for an area of $180 \mathrm{~nm} \times 120$ $\mathrm{nm}$.

The main benefit of our method comes from the fact that we work at a resonator (angular) frequency $\omega_{0}$ much lower than the CPB level spacing $\Delta E$. In Ref. [9] it is shown that $\omega_{0}$ depends on the resonator - CPB (qubit) interaction because of two contributions. The frequency change is $\Delta \omega_{0}=g^{2} / \delta$, where the detuning $\delta=\Delta E-\hbar \omega_{0}$, and the coupling coefficient $g$ contains the curvature of energy bands. In general, both $\delta$ and the curvature depend on the $\left(n_{g}, \varphi\right)$ point. Now, in our case everywhere $\Delta E \gg \hbar \omega_{0}, \delta \simeq \Delta E$, and hence $\Delta \omega_{0}=g^{2} / \Delta E=C_{\text {eff }} \omega_{0} /\left(2 C_{S}\right)$ has a contribution by only the second derivative, not by the detuning. Therefore, we can resolve the reactive response due to purely the bands of $\mathrm{CPB}$, which has not been possible in previous experiments.

When doing microwave spectroscopy, we have to consider also the other side of the coin: $\Delta E$ increases due to interaction with the resonator by 16$] \varepsilon=$ $\hbar\left(2 N g^{2} / \Delta E+g^{2} / \Delta E\right)$, where $N$ is the number of quanta in the resonator. When driven by a gate amplitude $V_{g}$, the resonator energy is $E_{R}=V_{g}^{2} C_{S} / 2$. At a high excitation amplitude $n_{g} \simeq 1 / 2$ we would have $V_{g} \simeq$ $e /\left(2 C_{g}\right)$ and hence $N=E_{R} /\left(\hbar \omega_{0}\right)=e^{2} C /\left(8 C_{g}^{2} \hbar \omega_{0}\right) \sim$ $4 \times 10^{3}$ which would yield $\varepsilon \sim \Delta E$. The data shown in this paper are, however, measured at a very low excitation of $n_{g} \sim 0.05$ which corresponds to $N \sim 40$ and $\varepsilon \sim 200 \mathrm{MHz}$ which is an insignificant contribution to $\Delta E$.

Fig. 2 (a) displays the measured phase shift $\Theta$ as a function of the two external control knobs (in the following, $n_{g}$ should be understood as being due to the control gate, $\left.n_{g}=C_{g 0} V_{g 0} / e\right)$. The results show full $2 e$ periodicity as a function of $n_{g}$, checked by increasing temperature above the $2 e-e$ crossover at $\sim 300 \mathrm{mK}$, and a $\Phi_{0}$ period with respect to $\Phi$. The data was measured without any microwave excitation, and hence we expect to see effects due to the ground band $C_{\text {eff }}^{0}$. The corresponding theoretical picture, obtained using Eq. (11) and straightforward circuit formulas for $\Gamma$, is given in Fig. 2 (b).

As a vital step to get convinced of the measured capacitance modulation versus the calculation, we carried out a detailed determination of the sample parameters independently of the capacitance modulation by using microwave spectroscopy (Fig. 3). To the weakly coupled control gate $C_{g 0}$ of the SCPT, we applied continuouswave microwaves while slowly sweeping the CPB band gap $\Delta E$ with $\varphi$ and $n_{g}$. Whenever the microwave energy 

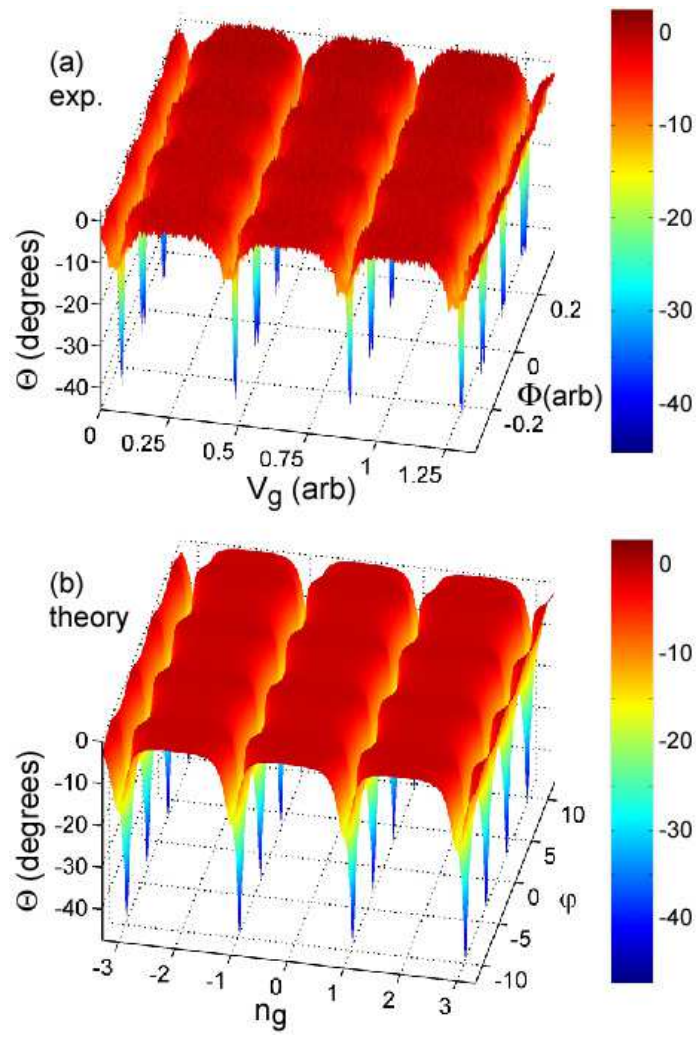

FIG. 2: (color online) (a) Phase shift $\Theta$ measured at a probing frequency $803 \mathrm{MHz} \sim f_{0}$, and (b) $\Theta$ calculated using Eq. (1) with the ground band $E_{0}\left(\varphi, n_{g}\right)$ evaluated numerically with parameters of Table 【

matches the band gap, that is, $\hbar \omega_{\mathrm{RF}}=\Delta E$, the CPB becomes resonantly excited. Since typically the Josephson corrections to the geometric capacitance are opposite in sign for the bands 0 and 1 (see Eq. (2)), band 1 would contribute an opposite phase shift signal. At resonance, we would then expect to see mixture of $C_{\text {eff }}^{0}$ and $C_{\text {eff }}^{1}$, weighted by the state occupancies which depend on the microwave amplitude. We calculate that a high enough amplitude sufficient to saturate the populations into a $50-50 \%$ mixture, would yield $\mathrm{a} \sim 3^{\circ}$ resonance absorption peak in the measured $\Theta$. The expectation is confirmed in Fig. B (b), where the resonance peaks are displayed at a few values of $\varphi$ (when $\varphi=0$, microwave energy does not exceed the band gap, and for $\varphi=\pi$ the peak height is lower due to a smaller matrix element).

While slowly sweeping $\varphi$ and $n_{g}$, the resonance conditions correspond to contours (see Fig. 3 (a)), which appear as annular ridges in the experimental data of graphs 3 (c)-(e) around the minimum $\Delta E$ at $\left(n_{g}=-1, \varphi=\pi\right)$. Since the band gap is sensitive to $E_{J}$ as well as to the $E_{J} / E_{C}$ ratio, the resonance contours allow for an accurate determination of these parameters (Table【). For example, at $\left(n_{g}=-1, \varphi=0\right)$, the band gap is $2 E_{J}=12.5$ $\mathrm{GHz}$, whereas at $\left(n_{g}=-1, \varphi=\pi\right) \Delta E$ has the absolute minimum $2 d E_{J} \simeq 3 \mathrm{GHz}$ which was barely exceeded by the microwave energy in Fig. 3 (c).

Based on the surface area $\sim 0.022(\mu \mathrm{m})^{2}$ of the overlay gate, we estimate $C_{g} \sim 0.5-1 \mathrm{fF}$. The exact value was obtained by fitting to the modulation depth of $C_{\text {eff }}^{0}$ (see Eq. (2)), yielding $C_{g}=0.65 \mathrm{fF}$, corresponding to a specific capacitance very reasonable to a thick oxide $\sim 30$ $\mathrm{fF} / \mu \mathrm{m}^{2}$.

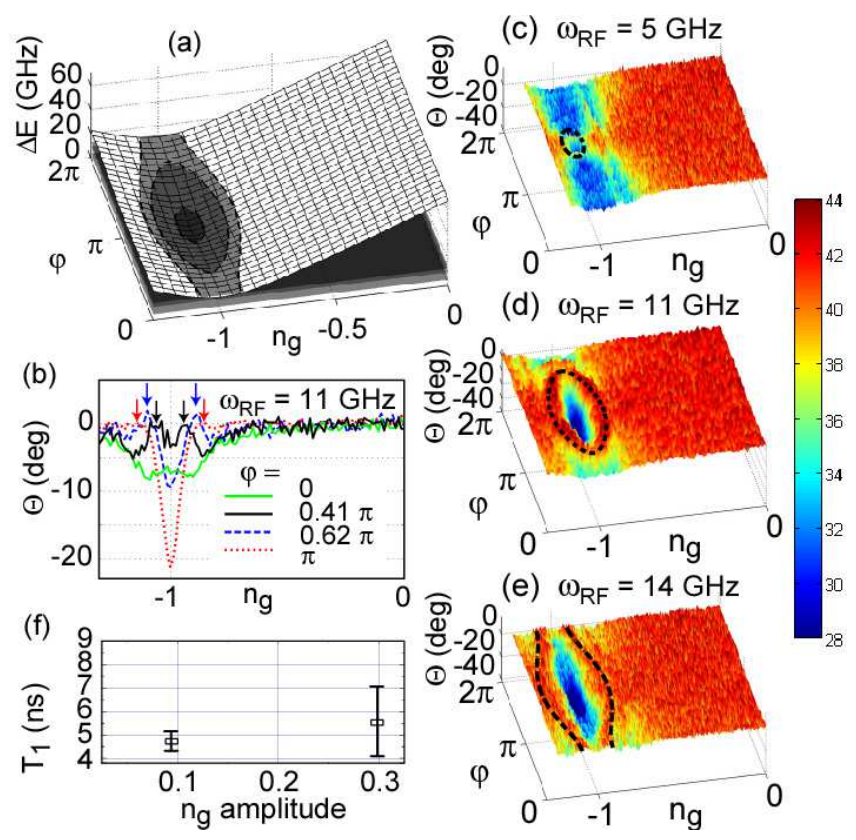

FIG. 3: (color online) (a) Illustration of the microwave spectroscopy used to map the SCPT band gap $\Delta E=E_{1}-E_{0}$. The three horizontal planes which intersect the band gap correspond, from bottom to top, to the microwave energy $\hbar \omega_{R F}$ used in (c)...(e), respectively. Whenever $\Delta E=\hbar \omega_{R F}$ (dashed lines), the system experiences resonant absorption; (b) peaks of resonant absorption (arrows) in the measured phase shift at $\omega_{R F}=11 \mathrm{GHz} ;(\mathrm{c})$ - (e) spectroscopy data represented as surfaces in the $\varphi, n_{g}$ plane. The resonance conditions shown in (a) are plotted on top of the data; (f) $T_{1}$ as a function of measurement strength at $\varphi=0, n_{g} \sim 1$.

\begin{tabular}{|c|c|c|c|c|c|c|}
\hline$E_{J}(\mathrm{~K})$ & $E_{C}=\frac{e^{2}}{2 C}(\mathrm{~K})$ & $E_{J} / E_{C}$ & $R_{T}(\mathrm{k} \Omega)$ & $C(\mathrm{fF})$ & $d$ & $C_{g}(\mathrm{fF})$ \\
\hline 0.30 & 0.83 & 0.36 & 55 & 1.1 & 0.22 & 0.65 \\
\hline
\end{tabular}

TABLE I: Sample parameters determined by microwave spectroscopy. $R_{T}$ is the series resistance of the two SCPT tunnel junctions (other parameters are defined in text and in Fig. 1).

Fig. (4 illustrates the bare gate and flux modulations without microwave excitation in more detail, and shows the corresponding numerical calculations using the ground band. As expected, $C_{\text {eff }}$ reduces to the geometric capacitance when Cooper-pair tunneling is blocked either by tuning the Josephson energy effectively to zero when $\varphi$ is an odd multiple of $\pi$, or by gate voltage. At the Coulomb resonance $n_{g}= \pm 1$, however, the Josephson capacitance is significant. In the special point $n_{g}= \pm 1$, 
$\varphi= \pm \pi$, the most pronounced effect is observed, now due to strong Cooper-pair fluctuations. The agreement between theory and experiment is good in Fig. 4 except around $n_{g}= \pm 1$ which we assign to intermittent poisoning by energetic quasiparticles [17]. An estimate using $C_{\text {eff }}$ from Eq. (2), $\Theta=-2 C_{\text {eff }} \sqrt{L} /\left(C_{S}^{3 / 2} Z_{0}\right)$, falls to within $15 \%$ of the numerical results except around integer $n_{g}$.
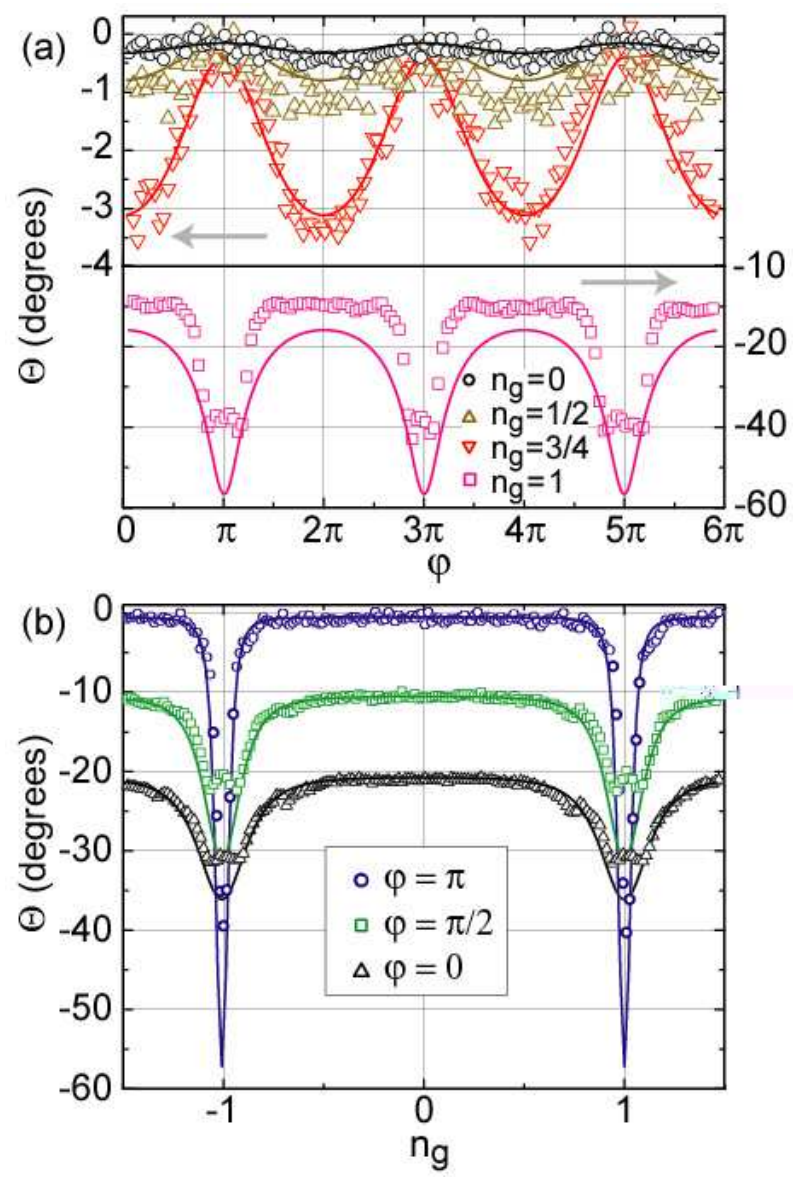

FIG. 4: (color online) Measured microwave phase shift $\Theta$, (a) vs. phase $\varphi$ (note different scales in the two panels) and (b) vs. gate charge $n_{g}$ (curves for $\varphi=0$ and $\pi / 2$ have been shifted vertically for clarity by $20^{\circ}$ and $10^{\circ}$, respectively). Solid lines are numerical calculations using Eq. (1) and sample parameters in Table I

Reactive measurements, either inductive or capacitive, can be employed for non-demolition readout for qubits 18] which means that $0 \leftrightarrow 1$ relaxation caused by the measurement is insignificant. An important advantage of our scheme is that since the probing gate swing has a frequency $f_{0} \ll \Delta E / \hbar$, its contribution to spectral density at the qubit level spacing frequency is negligible. We measured the relaxation time $T_{1}$ using the technique of pulsed microwave excitation with variable repetition time $T_{R}=1-200 \mathrm{~ns}$, while keeping the measurement signal always on, as in Ref. 19]. The $T_{1}$ times were limited to about 7 ns by parasitic reactances in the somewhat uncontrolled high-frequency environment, causing noise from $Z_{0}$ to couple strongly due to a large coupling $\kappa=C_{g} / C \sim 1$. The result for $T_{1}$, however, did not depend on the measurement strength (Fig. 3 (f)), which supports the non-demolition character of this scheme. By fabricating the resonator on-chip it is straightforward to gain a full control of environment. Then, the impedance seen from the qubit gate $\operatorname{Re}\left(Z_{g}(\omega=\Delta E / \hbar)\right) \simeq 0.1 \mathrm{~m} \Omega$, and a worst-case estimate yields $T_{1} \approx \hbar R_{K} /\left[4 \pi \kappa^{2} \operatorname{Re}\left(Z_{g}(\Delta E / \hbar)\right) \Delta E\right] \gg 1 \mu \mathrm{s}$. For a dephasing time $T_{2}$ averaged over $n_{g}$, we measured $\sim 0.5$ ns using Landau-Zener interferometry [20, 21]. This $T_{2}$ time is on the same order as the spectroscopy line widths in Fig. 3 .

In conclusion, using the phase of strongly reflected microwave signals, we have experimentally verified the Josephson capacitance in a mesoscopic Josephson junction, i.e., the quantity dual to the Josephson Inductance. Good agreement is achieved with the theory on the Josephson capacitance. Implications for non-destructive readout of quantum state of Cooper-pair box using the capacitive susceptibility are investigated.

We thank T. Heikkilä, F. Hekking, G. Johansson, M. Paalanen, and R. Schoelkopf for comments and useful criticism. This work was supported by the Academy of Finland and by the Vaisala Foundation of the Finnish Academy of Science and Letters.

[1] M. Tinkham, Introduction to Superconductivity (McGraw-Hill, New York, 1996).

[2] See, e.g., G. Schön and A.D. Zaikin, Phys. Rep. 198, 237 (1990); J.S. Penttilä et al., Phys. Rev. Lett. 82, 1004 (1999).

[3] Y. Nakamura, Y. A. Pashkin, and J. S. Tsai, Nature 398, 786 (1999).

[4] A. Widom et al., J. Low Temp. Phys. 57, 651 (1984).

[5] D. V. Averin, A. B. Zorin, and K. Likharev, Sov. Phys. JETP 61, 407 (1985); K. Likharev and A. Zorin, J. Low. Temp. Phys. 59, 347 (1985).

[6] D. V. Averin and C. Bruder, Phys. Rev. Lett. 91, 057003 (2003).

[7] See, e.g., Yu. Makhlin, G. Schön, A. Shnirman, Rev. Mod. Phys. 73, 357 (2001).

[8] Capacitance of a non-equilibrium Cooper pair box has been investigated in a recent preprint; T. Duty et al., cond-mat/0503531

[9] A. Wallraff et al., Nature 431, 162 (2004).

[10] The last term in the Hamiltonian typically does not influence the SCPT's dynamics and is typically omitted; it is important to keep it here since $V_{g}$ becomes a dynamical variable.

[11] L. D. Landau and E. M. Lifshitz, Mechanics (Pergamon Press, Oxford, 1976).

[12] For single electron case, see M. Büttiker and C. A. Stafford, Phys. Rev. Lett. 76, 495 (1996). 
[13] M.A. Sillanpää, Ph.D. thesis, Helsinki University of Technology (2005); http://lib.tkk.fi/Diss/2005/isbn9512275686/

[14] M. Sillanpää, L. Roschier, and P. Hakonen, Phys. Rev. Lett. 93, 066805 (2004).

[15] L. Roschier, M. Sillanpää, and P. Hakonen, Phys. Rev. B 71, 024530 (2005).

[16] D. I. Schuster et al., Phys. Rev. Lett. 94, 123602 (2005).

[17] J. Aumentado, M. W. Keller, J. M. Martinis, and M. H.
Devoret, Phys. Rev. Lett. 92, 066802 (2004).

[18] A. Blais et al., Phys. Rev. A 69, 062320 (2004).

[19] T. Duty, D. Gunnarsson, K. Bladh, and P. Delsing Phys. Rev. B 69, 140503 (2004).

[20] A. V. Shytov, D. A. Ivanov, and M. V. Feigel'man, Eur. Phys. J. B 36, 263 (2003).

[21] M. A. Sillanpää et al., cond-mat/0510559 